\title{
The influence of micrometastases on prognosis and survival in stage I-II colon cancer patients: the Enroute $\oplus$ Study
}

Daniel J Lips ${ }^{1 *}$, Boukje Koebrugge ${ }^{1,2}$, Gerrit Jan Liefers², Johannes C van de Linden ${ }^{3}$, Vincent THBM Smit ${ }^{4}$, Hans FM Pruijt ${ }^{5}$, Hein Putter ${ }^{6}$, Cornelis JH van de Velde ${ }^{2}$ and Koop Bosscha ${ }^{1}$

\begin{abstract}
Background: The presence of lymph node metastases remains the most reliable prognostic predictor and the gold indicator for adjuvant treatment in colon cancer (CC). In spite of a potentially curative resection, 20 to 30\% of CC patients testing negative for lymph node metastases (i.e. pNO) will subsequently develop locoregional and/or systemic metastases within 5 years. The presence of occult nodal isolated tumor cells (ITCs) and/or micrometastases (MMs) at the time of resection predisposes CC patients to high risk for disease recurrence. These $\mathrm{pNO}_{\text {micro+ }}$ patients harbouring occult micrometastases may benefit from adjuvant treatment. The purpose of the present study is to delineate the subset of pNO patients with micrometastases ( $\mathrm{pNO}_{\text {micro+ }}$ ) and evaluate the benefits from adjuvant chemotherapy in $\mathrm{pNO}_{\text {micro+ }} \mathrm{CC}$ patients.

Methods/design: EnRoute+ is an open label, multicenter, randomized controlled clinical trial. All CC patients (age above 18 years) without synchronous locoregional lymph node and/or systemic metastases (clinical stage I-II disease) and operated upon with curative intent are eligible for inclusion. All resected specimens of patients are subject to an ex vivo sentinel lymph node mapping procedure (SLNM) following curative resection. The investigation for micrometastases in pNO patients is done by extended serial sectioning and immunohistochemistry for pan-cytokeratin in sentinel lymph nodes which are tumour negative upon standard pathological examination. Patients with ITC/MM-positive sentinel lymph nodes ( $\mathrm{pNO}_{\text {micro++ }}$ ) are randomized for adjuvant chemotherapy following the CAPOX treatment scheme or observation. The primary endpoint is 3-year disease free survival (DFS).
\end{abstract}

Discussion: The EnRoute+ study is designed to improve prognosis in high-risk stage I/II pNO micro+ CC patients by reducing disease recurrence by adjuvant chemotherapy.

Trial Registration: ClinicalTrials.gov: NCT01097265

\section{Background}

Colorectal cancer is the second most commonly diagnosed malignancy in men and women in the Netherlands with increasing incidence due to growth and ageing of the general population [1]. The presence of lymph node metastases remains the most reliable prognostic predictor and the gold indicator for adjuvant treatment in colon cancer (CC) [2]. Interestingly, in a large percentage of patients without lymph node

\footnotetext{
* Correspondence: djlips@home.nl

'Department of Surgery, Jeroen Bosch Hospital, Nieuwstraat 34, 5211 NL 'sHertogenbosch, the Netherlands
}

Full list of author information is available at the end of the article metastases in the surgical specimen, who are therefore not subjected to adjuvant chemotherapy, represent with disease recurrence. In about $10 \%$ of the patients with stage I (Dukes A) and 15-30\% with stage II (Dukes B) disease recurrent locoregional or distant metastases develop within 5 years [2-4]. One possible factor could be the presence of occult lymph node metastases at the time of presentation and surgical resection. Evidence has emerged showing a significant amount of nodal metastases being smaller than $2 \mathrm{~mm}$ or less $(<0.2 \mathrm{~mm}$ isolated tumor cells [ITC]; $0.2-2 \mathrm{~mm}$ micrometastasis $[\mathrm{MM}]$ ) and, therefore, likely to be missed during conventional gross pathological specimen examination.
C Biomed Central

(c) 2011 Lips et al; licensee BioMed Central Ltd. This is an Open Access article distributed under the terms of the Creative Commons Attribution License (http://creativecommons.org/licenses/by/2.0), which permits unrestricted use, distribution, and reproduction in any medium, provided the original work is properly cited. 
[3-5] Focused examination methods, such as more extensive nodal examination by serial sectioning or step sectioning, or molecular detection of metastatic nodal cells by immunohistochemistry (IHC) or reverse transcriptase-polymerase chain reaction (RT-PCR), increase the likelihood of finding these tumour deposits. However, these focused examination methods are expensive and time consuming, and therefore not applicable to all lymph nodes derived from the surgical specimen. By using sentinel lymph node mapping (SLNM) the nodes at highest risk of harbouring tumour deposits can be potentially detected and more thoroughly examined. The ex vivo SLNM procedure is technically easy, as the procedure is executed extracorporally by injection peritumoral blue dye subserosally or submucosally after which gentle massage of the injection site is performed [5-11]. Blue coloured lymph nodes are excised or marked by sutures. The ex vivo SLNM procedure is characterized by a high accuracy of $90-100 \%$, and negative predictive value of $80-100 \%$ [6-11]. A rate of $19-57 \%$ upstaging is observed [6-11].

Current knowledge about the prognostic relevance of nodal micrometastases and isolated tumor cells has adequately been reviewed in 2004 separately by Iddings and Nicastri $[3,4]$. They concluded that a suggestion of prognostic relevance could be made for micrometastatic disease related to worsened disease-free survival (DFS) and overall survival (OS). The meta-analysis performed by Iddings showed a decreased 3-year DFS and OS of respectively $78 \%$ and $78 \%$ in $\mathrm{pNO}_{\text {micro+ }}$ patients compared to $90 \%$ and $97 \%$ in $\mathrm{pNO}_{\text {micro- }}$ patients [4]. New evidence from two large international prospective observational studies shows a clear negative prognostic effect of micrometastatic nodal disease [5]. The 4-year DFS detoriated from $94 \%$ to $78 \%$ with the presence of nodal micrometastasis [5]. A solid conclusion, however, could not be made because of the lack of well-designed, well powered, controlled clinical trials. Data from other solid organ malignancies like breast cancer links micrometastatic disease to a worsened prognosis [12]. Several prospective trials are currently recruiting (see table 1 ). However, an randomized, controlled clinical trial of significant magnitude is needed to answer this clinically relevant question.

\section{Table 1 Currently recruiting studies}

\begin{tabular}{lcl}
\hline $\begin{array}{l}\text { University Hospital, Strasbourg, France } \\
\text { (C. Brigand) }\end{array}$ & $2007-2014$ & $\mathrm{~N}=140$ \\
$\begin{array}{l}\text { Jonsson Comprehensive Cancer Center } \\
\text { (A. Bilchik) }\end{array}$ & $2009-2011$ & $\mathrm{~N}=300$ \\
$\begin{array}{l}\text { John Wayne Cancer Institute (S. Baker) } \\
\text { University Hospital, Basel, Switzerland } \\
\text { (C. Viehl) }\end{array}$ & $2000-2011$ & $\mathrm{~N}=200$ \\
\hline
\end{tabular}

Source: http://www.clinicaltrials.gov
Nowadays, adjuvant chemotherapy is only offered to high risk stage I-II colon cancer patients in the Netherlands [2]. Stage I-II CC patients without risk factors are thought not to benefit from adjuvant treatment. Individual randomized trials did not show a significant survival benefit in stage II colon cancer patients [2]. The results of meta-analyses and systematic reviews show at the most a slight disease-free survival benefit of adjuvant chemotherapy in stage II disease [2]. However, to stress out the importance of further investigation, stage I-II $\mathrm{CC}$ patients do suffer from disease recurrence and their overall 5 -year survival is just around $70-80 \%$. Even stage II CC patients without risk factors have been shown in several studies to benefit from adjuvant treatment $[13,14]$. It is because of these results that in Eastern countries and the United States stage II CC patients with or without micrometastatic disease do receive adjuvant treatment. Thus, there is an international need for better delineation of high-risk stage I-II CC patients who probably should be offered adjuvant treatment.

\section{Methods/Design \\ Study objectives}

To determine the benefits from adjuvant chemotherapy in $\mathrm{pNO}_{\text {micro+ }} \mathrm{CC}$ patients on 3-year disease-free survival compared to non-treated $\mathrm{pNO}_{\text {micro+ }} \mathrm{CC}$ patients.

\section{Primary endpoint}

Primary endpoint is 3-year DFS in study groups (proportion of patients without local or distant recurrence, or second primary of same of other cancer, death from same cancer of any other cause during the defined period of time).

\section{Secondary endpoints}

Secondary endpoints are rate of upstaging in pNO colonic cancer patients (total number $\mathrm{pNO}_{\text {micro+ }}$ patients $\times$ 100/total number pN0 colon cancer patients) and 3-year overall survival.

\section{Design}

EnRoute+ is an open label, multicenter, randomized controlled clinical trial. A centrally-performed, computer-generated randomization procedure is instituted. Eligible patients are randomly assigned at a 1:1 ratio to receive treatment respectively without or with chemotherapy using block-randomisation per participating center. Design, flow chart and follow up are presented in Figures 1, 2 and 3.

Study groups are defined according the randomisation group and pathological examination result.

group A $\mathrm{pNO}_{\text {micro+ }}$ with chemotherapy

group $\mathrm{B} \quad \mathrm{pNO}_{\text {micro+ }}$ without chemotherapy

group $\mathrm{C} \mathrm{pNO}_{\text {micro- }}$ without chemotherapy 


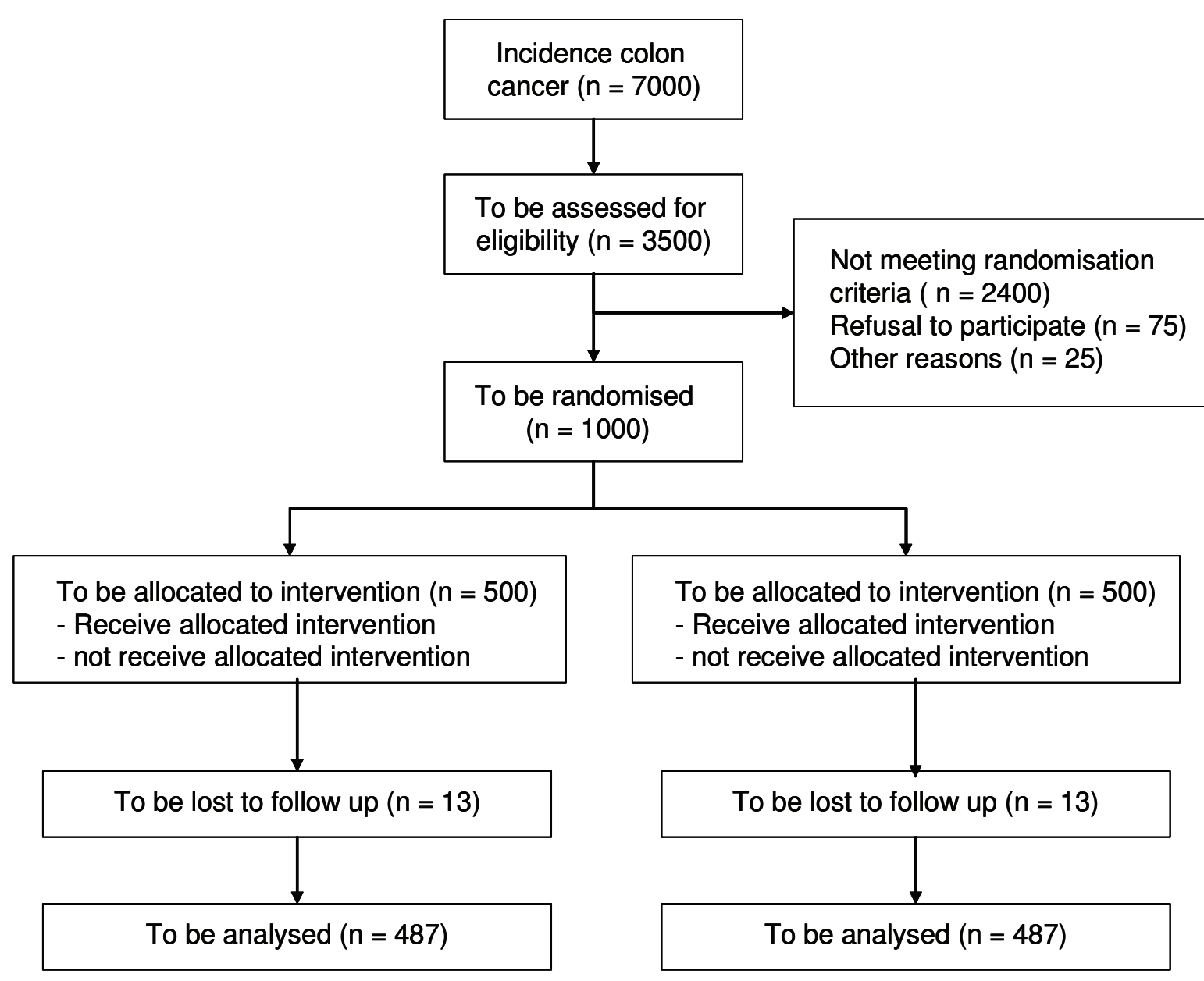

Figure 1 EnRoute+ study according to CONSORT

\section{Setting}

Subjects will be enrolled in multiple academic and nonacademic (teaching and non-teaching) hospitals in the Netherlands.

\section{Patients}

A total of 1000 adult patients of both sexes with $\mathrm{pNO}_{\text {mi- }}$ cro+ will be randomized to receive adjuvant chemotherapy.

\section{Eligibility criteria}

\section{Inclusion criteria for randomization}

- $\mathrm{pNO}_{\text {micro+ }}$ colon cancer patients (stage I/II, Dukes $\mathrm{A} / \mathrm{B})$

- patients deemed to be fit for chemotherapy treatment (WHO classification $\leq 1$; ASA classification $\leq$ 2)

\section{Exclusion criteria for randomization}

- rectal cancer
- previous chemotherapy

- high risk pN0 patient according to:

o less then 10 lymph nodes detected in resected specimen, or

$\circ$ invasion in other organs (T4NxMx), or

$\circ$ colon perforation at presentation, or

$\circ$ clinically relevant obstruction at presentation, or

$\circ$ angioinvasion at conventional pathological examination

- positive nodal involvement or advanced disease (stage III/Dukes C/Dukes D)

- haematological impairments (anemia, leucopenia, thrombocytopenia within 7 days before start of chemotherapy treatment)

$\circ$ renal function impairments (creat.clearance $\leq$ $60 \mathrm{ml} / \mathrm{min}$ within 7 days before start of chemotherapy treatment)

- liver enzyme serum alterations within 7 days before start of treatment 


\section{EП ROUTE $\oplus$ STUDY}

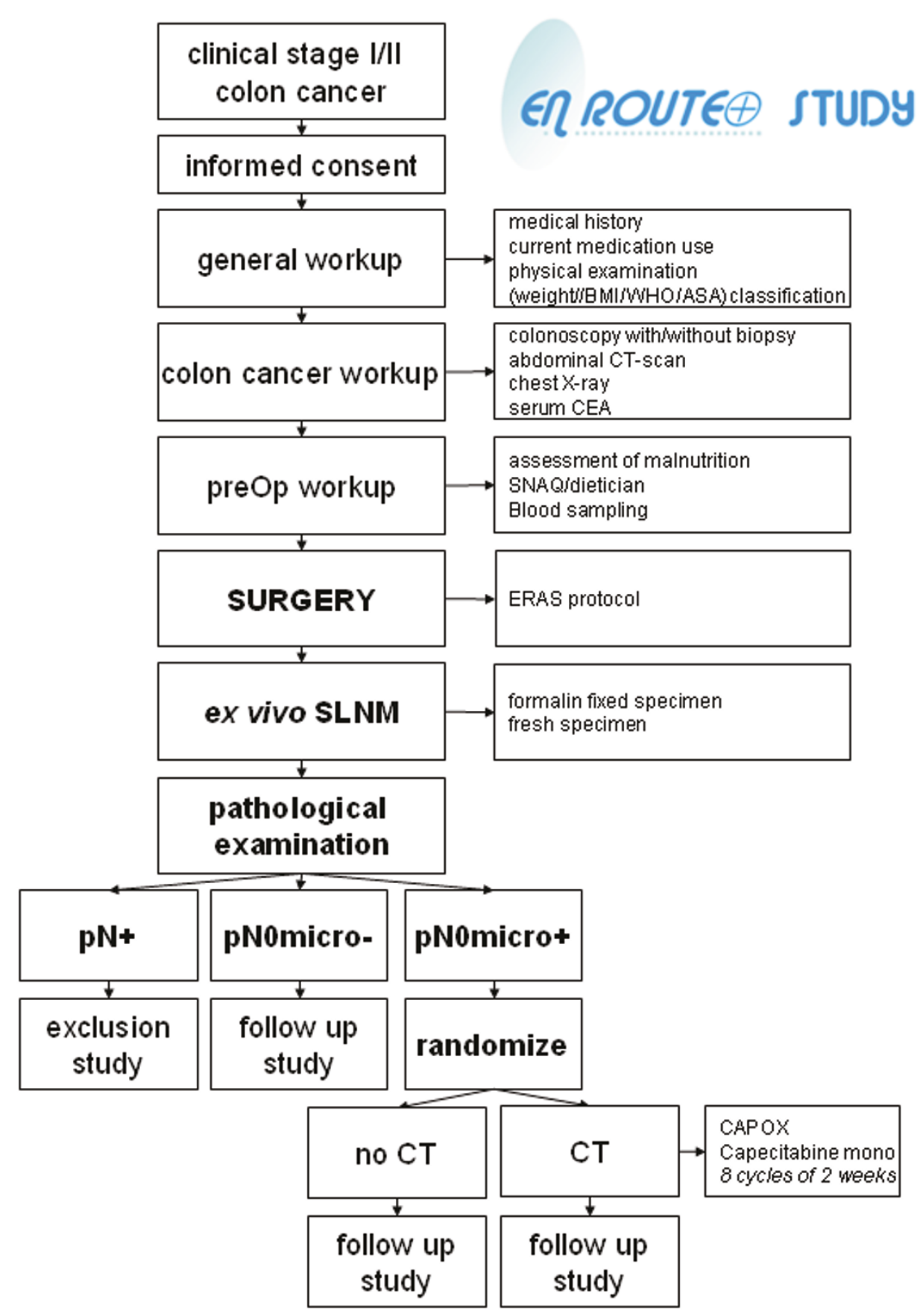

Figure 2 EnRoute+ flow chart

- other current serious illness or medical conditions

o severe cardiac illness (NYHA class III-IV, see

Appendix)

o significant neurologic or psychiatric disorders

$\circ$ uncontrolled infections

$\circ$ active disseminated intravascular coagulation $\circ$ other serious underlying medical conditions that could impair the ability of the patient to participate in the study

- definite contraindications for the use of corticosteroids

- use of immunosuppressive or antiviral drugs 


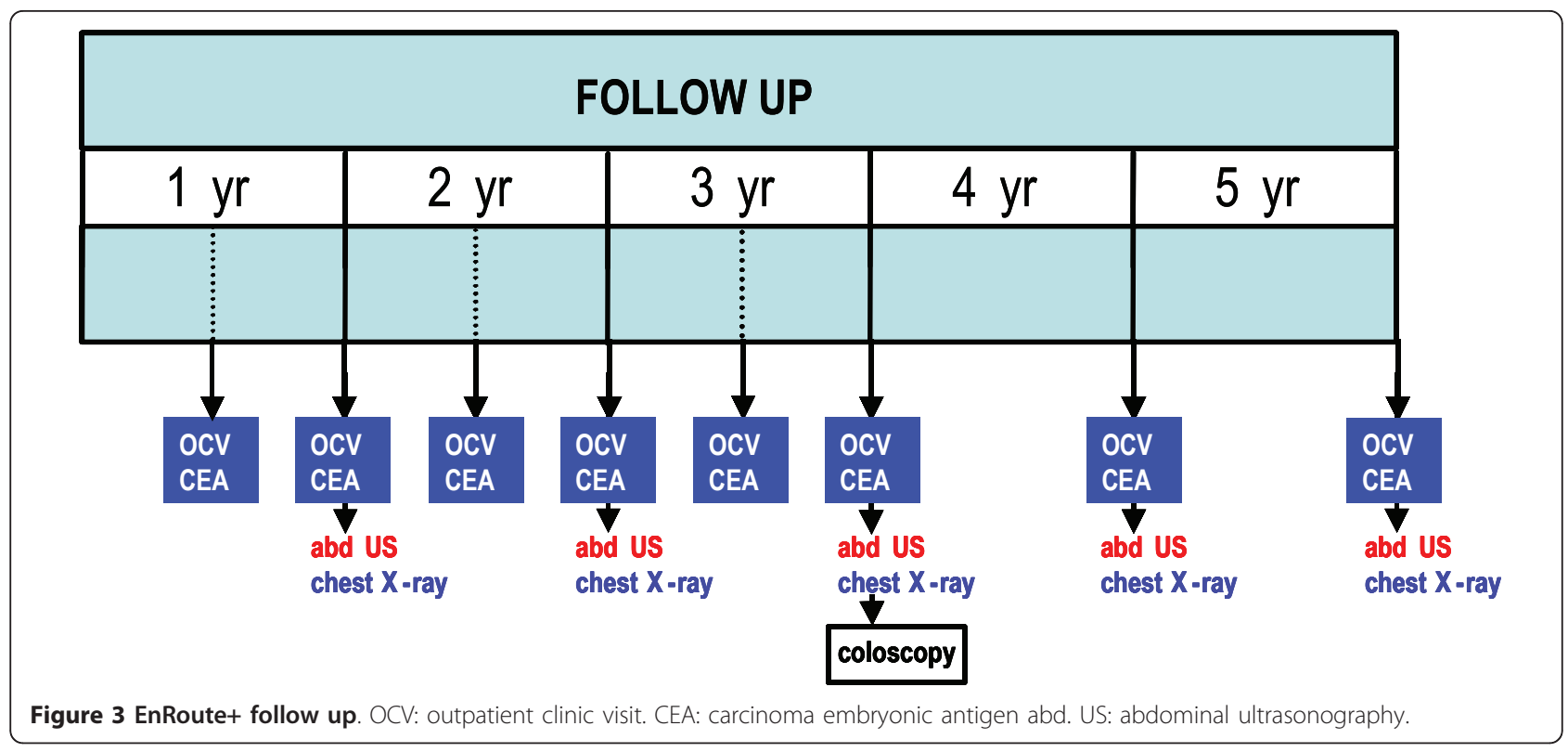

- any other experimental drugs within a 4-week period prior to start of surgery and adjuvant chemotherapy and/or throughout/during the study period

- pregnancy or lactation

- patients unable to comply with requirements of the study

\section{Ethics, informed consent}

This study is conducted in accordance with the principles of the Declaration of Helsinki and 'good clinical practice' guidelines. The independent central ethics committee approved the final protocol on $26^{\text {th }}$ July 2010. Oral and written informed consent is obtained from the patient before inclusion in the trial.

\section{Safety}

No additional risks for patients are introduced by the $e x$ vivo SLNM procedure as the complete procedure is performed extracorporally. Participation in the randomization study brings risks and discomfort for the patient common to adjuvant systemic chemotherapeutic treatment as given to patients with stage III colon cancer (CAPOX scheme). Dose limiting toxicities of oxaliplatin/ capecitabine include diarrhoea, abdominal pain, nausea, stomatitis and hand-foot syndrome (hand-foot skin reaction, palmar-plantar erythrodysesthesia). Most adverse reactions are reversible and do not require permanent discontinuation of therapy, although doses may need to be withheld or reduced. A potential negative effect on prognosis can be expected in patients not receiving adjuvant chemotherapy.

\section{Statistical analysis}

\section{Intention-to-treat}

The analysis will be performed on the basis of an intention-to-treat (ITT) population and with respect to ITT principles. Also a per-protocol (chemotherapy versus no adjuvant treatment) analysis will be performed.

\section{Interim analysis}

An interim analysis will be performed when half of the required number of events have been observed. Based on the O'Brien-Fleming alpha-spending function, the first analysis will be performed at nominal alpha level 0.003, the second at nominal alpha level 0.047, to ensure an overall alpha of 0.05 .

\section{Sample size}

The sample size is calculate to be $(\mathrm{N}=) 973$ patients (control group [B] 486 pts/145 events, treatment group [C] 487 pts/102 events with $90 \%$ power and a significance level of 0.05 . Loss to follow up is expected to be 26 patients. Median follow up for primary outcome is 3 years. Accrual rate is expected to be at least 300 patients/year, which results in a maximum total accrual time of 4 years. Median follow up of 3 years is reached after 5 years total study time.

\section{Treatment program}

Eligible patients are selected and informed by local clinicians. Written consent is obtained and the patient is prepared for surgery according to the ERAS protocol. The method of surgery (i.e. laparoscopic or open) is chosen by the surgeon together with the patient. The datacenter is informed by the treating clinician about registration of the patient into the trial and the preoperative CRF is send to the datacenter. The operation is performed by a certified surgeon, or supervised surgical resident. Surgery 
is performed according to anatomical and oncological principles dictated by the location of the tumour. The adjacent mesocolon is dissected including its base and excised en-block. Within fifteen minutes of the resection the ex vivo SLNM is performed. The specimen is opened at the anti-mesenteric side. When the tumour is identified, $1 \mathrm{ml}$ of Patent Blue V dye is injected into the submucosa circumferentially to the tumour, using a tuberculin syringe. The injection sites are then gently massaged for up to five minutes to push the tracer into the lymphatic vessels. Blue coloured sentinel nodes are identified and marked with a suture. The specimen is transferred fresh to the pathology laboratory, when possible according to local pathological facilities, for further examination and processing. The specimen is put in formalin within 15 minutes after the SLNM procedure, when local pathological facilities dictate formalin embedded transportation of the specimen. If fresh received, a sample is taken from the primary tumor for tumor banking and side-study purposes. The pathologist examines the specimen using conventional methods and identifies the blue stained nodes and puts them into marked cassettes so that they can be examined by microscopic examination. Sentinel and non-sentinel lymph nodes are bivalved. Each section is analyzed using conventional histologic staining with hematoxylin and eosin (H\&E). Efforts are made to analyze at least 10 lymph nodes per specimen. Detection of lymph node metastases is performed by microscopic examination in sentinel and non-sentinel nodes. The patient is excluded for focussed examination, when macroscopic metastases are present $(\mathrm{pN}+)$ or angioinvasion is detected (see exclusion criteria). In pN0 patients sentinel lymph nodes are additionally sliced at four levels at $150 \mu \mathrm{m}$ intervals. All sections are stained with Pan-Cytokeratin (LU-5). Positive cytokeratin lymph node metastases are defined as ITC $<0.2$ mm or MM $0.2-2$ mm (see Table 2). Rare single cells staining positive with IHC that lack cytologic characteristics of malignancy are considered tumor-negative. Tumours are staged according to the AJCC TNM classification 2002 (Sixth Edition). Adjuvant chemotherapy is provided according to the CAPOX (XELOX) protocol

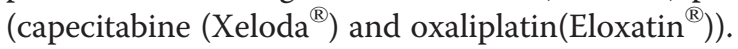

\section{Monitoring}

Research nurses monitor the participating centres and patients. Every 6 months a random selection of participating centres is visited by research nurses checking, at least, $10 \%$ of patient's data.

\section{Follow-up}

Patients are followed during their hospital stay. Patients are kept in follow up for five years according to national guidelines (see Figure 3) [2].

\section{Discussion}

Recurrent locoregional and/or systemic disease in stage I/II colon cancer is a significant clinical and social health care problem. The fact of high disease recurrence in stage I-II colon cancer patients is the basis of the study idea and the justification for the study. Around 7000 new colon cancer patients are diagnosed yearly in the Netherlands at this moment and the incidence will rise in the coming decades [2]. Of these new colon cancer patients respectively 16 and $38 \%$ have stage I or stage II disease [15]. Therefore, yearly approximately 3500 new stage I-II patients are at risk for disease recurrence. A upstaging rate of $30 \%$ is expected to be observed by using SLNM, serial sectioning and IHC for micrometastatic disease detection [6-11]. Approximately 1050 are at high risk for disease recurrence. Nowadays, adjuvant chemotherapy is only offered to high risk stage II colon cancer patients in the Netherlands [2]. Stage II CC patients without risk factors are thought not to benefit from adjuvant treatment. Individual randomized trials did not show a significant overall survival benefit in stage II colon cancer patients [2]. The results of meta-analyses and systematic reviews show at the most a slight disease-free survival benefit of adjuvant chemotherapy in stage II disease of $3-6 \%$ absolute risk reduction in disease-free survival (3.8 - 6\% ARR in 5-yr DFS by Figueredo et al, 3\% ARR in 5-yr DFS in the IMPACT-B2 study, 4\% ARR in 5-yr DFS in the Gillstudy [2,16-18].

According to SEER data 5-year overall survival data of stage IIa (T3N0M0), IIb (T4NOM0) and IIIa (T1$2 \mathrm{~N} 1 \mathrm{M} 0)$ and b (T3-4N1M0) are respectively $84.7 \%$,

Table 2 Definitions of isolated tumor cells (ITCs) and micrometastases (MMS)

\begin{tabular}{|c|c|c|}
\hline \multicolumn{3}{|l|}{ Definitions used in EnRoute $\oplus$ Study } \\
\hline & Isolated Tumor Cells (ITCs) & Micrometastases (MMs) \\
\hline Size & $<0.2 \mathrm{~mm}$ & $0.2-2 \mathrm{~mm}$ \\
\hline Localization & No defining criterion & No defining criterion \\
\hline Detection method & HES and/or IHC & HES and/or IHC \\
\hline Formal designation (present vs absent) & $\mathrm{pNO}_{\mathrm{i}+}$ vs $\mathrm{pNO}_{\mathrm{i}-}$ & $p N 0_{m i+} v s p N 0_{m i-}$ \\
\hline Designation for study purposes if present & $\mathrm{pNO}_{\text {micro+ }}$ & $\mathrm{pNO}_{\text {micro+ }}$ \\
\hline
\end{tabular}

HES hematoxylin and eosin stain; IHC immunohistochemistry. 
$72.2 \%, 83.4 \%$ and $64.1 \%$ [19]. The MOSAIC-trial observed a 4-year DFS of untreated versus treated colon cancer stage II and III of respectively $85-87.6 \%$ and 66.5-74.1\% [20]. The XELOXA-trial found a 3-year DFS of $66.5-70.9 \%$ (untreated versus treated) in stage III colon cancer [21]. An 9\% absolute risk reduction on 5year OS (85.4\% in the surgery-only group and $94.2 \%$ adjuvant chemotherapy group) was observed in T3N0M0 patients following adjuvant chemotherapy in a large retrospective study [13]. A similar result was observed in 468 stage II patients (i.e. 8\% 4-year OS risk reduction of $70 \%$ to $78 \%$ ) [22]. In an Australian population-based cohort-study patients with good prognosis (T3, no lymphovascular invasion) showed improved 5year survival rate with chemotherapy from 85 to 95\% (P $=0.064$, log-rank test) in women but showed no change in men (84 vs $82 \%$ ). The poor prognosis (T4 and/or presence of lymphovascular invasion) patients, survival rates for both women (65 vs 79\%) and men (72 vs 78\%) improved with the use of chemotherapy, however, did not reach significance in either group $(\mathrm{P}=0.22$ and $\mathrm{P}=$ 0.26 , respectively) [14]. It is therefore assumed that the use of adjuvant chemotherapy in high-risk pN0 colon cancer patients could result in clinical significant health benefits for individual patients.

The detection of sentinel nodes using a SLNM procedure has been extensively investigated [5-9,11,23-29]. For correct interpretation of these data a gross distinction has to be made between i) in vivo and ex vivo procedures; ii) immunohistochemical (IHC) or reversetranscriptase polymerase chain reaction (RT-PCR) occult nodal metastatic disease detection.

The in vivo SLNM procedure is characterized by a more difficult technical procedure, most notably during a laparoscopic colonic resection, as a subserosal dye injection is performed preoperatively [23-29]. The accuracy and negative predictive value of in vivo SLNM procedures are high and range from 90 to $95 \%$ and 93 to $97 \%$ respectively [23-29]. Upstaging was observed in 28-35\% of H\&E pN0 patients [23-29]. The ex vivo SLNM procedure is technically much easier, as the procedure is executed extracorporally by injection peritumoral blue dye subserosally or submucosally after which gentle massage of the injection site is performed [6-11]. The ex vivo SLNM procedure is characterized also by a high accuracy of $90-100 \%$, and negative predictive value of $80-100 \%$ [6-11]. A rate of $19-57 \%$ upstaging is observed [6-11].

Although most studies combine data of colon and rectal cancer, a clear distinction in sentinel lymph node mapping between both cancers has been observed in some well-designed studies $[6,11,26]$. The accuracy of negative predictive value of SLNM in rectal cancer is estimated to be as low as $76 \%$ and $65 \%$ respectively, and rectal cancers are therefore excluded from inclusion [6].
The method of examination, i.e. IHC or RT-PCR, is also an important determinant of outcome $[3,4]$. Cytokeratin is a cellular marker for epithelial origin, adhering to constituents of the cytoskeleton of both normal and malignant cells. However, epithelial cells are not normal constituents of lymphoid tissue, and morphological examination of all cytokeratin-positive cells for secondary malignant characteristics is mandatory. Most studies used an IHC methodology, with little similarity in immunohistochemical markers unfortunately $[3,4,6-9,24,30]$. A high variety in the use of molecular markers for RT-PCR inhibits the selection of the one with highest accuracy and negative predictive value $[3,4,23]$. The best correlation between micrometastatic disease and worsened DFS and OS has been observed in RT-PCR detected occult disease in comparison to IHC $[3,4]$. The strength of the evidence of this observed correlation is weak though. Furthermore, RNA is subtracted from halve of a sentinel node in RT-PCR procedures. This unables pathologists the examination of secondary malignant characteristics with $H \& E$ colouring. Moreover, the authors of the most recent prospective clinical trial using RT-PCR stated that quantified RT-PCR assays may be of value when cytokeratin immunohistochemistry fails to detect sentinel lymph node metastasis [23]. Therefore, no definitive preference for IHC or RT-PCR methods can be given. For practical arguments, including the possibility for all Dutch hospitals to participate in the study, a controlled clinical trial using an ex vivo SLNM procedure with focussed nodal examination by cytokeratin IHC techniques is proposed.

Adjuvant chemotherapy is provided according to the CAPOX (XELOX) protocol (capecitabine (Xeloda ${ }^{\circledR}$ ) and oxaliplatin(Eloxatin $\left.{ }^{(}\right)$). This CAPOX (XELOX) protocol is assumed to be comparable in therapeutical effect and toxicity to the currently used FOLFOX-4 protocol, and more patient friendly because of the oral supplementation of chemotherapy (capecitabine) [2].

As primary endpoint is 3-year DFS chosen as surrogate endpoint for overall survival. Colon cancer recurrence is most notably observed in the first years after initial treatment [2,31]. Furthermore, 3-year DFS is recognized as an appropriate surrogate end point for overall 5-year survival in colon cancer trials [32-34].

\section{Conclusion}

Around $30 \%$ of all pNo colon cancer patients will develop locoregional and/or distant disease recurrence. Adjuvant systemic chemotherapy is thought to decrease disease recurrence in high risk $\mathrm{pN}_{\text {micro+ }}$ patients. A large patient group is subject to this high disease recurrence rate. Therefore, it is justified to perform the proposed trial in a multicenter design in the Netherlands to answer the clinical relevant question of the role of 
micrometastatic disease on prognosis in stage I-II colon cancer patients.

\begin{abstract}
Abbreviations
CC: colon cancer; ITC: isolated tumor cell; MM: micrometastasis; SLNM: sentinel lymph node mapping; IHC: immunohistochemistry; RT-PCT: reverse transcriptase-polymerase chain reaction; DFS: disease-free survival; OS: overall survival; pNO: no lymph node metastasis on pathological examination; $\mathrm{pNO}_{\text {micro+/:: }}$ present/absent micrometastasis on pathological examination; pN +: lymph node metastasis on pathological examination; WHO: World Health Organisation; ASA: American Society of Anesthesiologists; NYHA: New York Heart Association; ITT: intention-to-treat; ERAS: enhanced recovery after surgery; CRF: case report form; H\&E: hematoxylin and eosin; CAPOX/XELOX: capecitabine $\left(X_{\text {eloda }}{ }^{\mathbb{}} \oplus\right.$ ) and oxaliplatin; FOLFOX: fluorouracil and oxaliplatin.
\end{abstract}

\section{Acknowledgements}

The EnRoute $\oplus$ Study is funded by the Dutch Cancer Society (grant-number 2010-4788) and by Roche Pharmaceuticals through an Educational Grant (grant-number ML25369). Protocol developed during the 12th joint ECCOAACR-EORTC-ESMO Workshop 'Methods in Clinical Cancer Research', Waldhaus Flims, Switzerland, $19-25$ June 2010 by D.J. Lips as a fellow of the European Society of Surgical Oncology.

\section{Ethics}

The study protocol is approved by the Dutch central medical-ethical body METOPP (Tilburg, the Netherlands) referred to as protocol number

\section{NL31438.028.10}

\section{EnRoute+ study committee members}

\section{Steering Committee and Key Staff at coordinating centres}

K. Bosscha (principal investigator), D.J. Lips (study coordinator), B. Koebrugge (Ph.D.-student), H. Pruijt, J.C. van der Linden, Jeroen Bosch Hospital; C.J.H. van de Velde (co-principal investigator), G.J. Liefers, V.T.H.B.M. Smit, H. Putter, LUMC.

\section{Clinical centres and investigators}

Jeroen Bosch Hospital, K. Bosscha

Leiden University Medical Centre, C.J.H. van de Velde

Viecuri Medical Centre, J.L.M. Konsten

Medical Spectrum Twente, J. M. Klaase

Academic Hospital Maastricht, M.L. Smidt

Sint Lucas Andreas Hospital, B.C. Vrouenraets

Diakonessen Hospital Utrecht, P.H.P. Davids

Amphia Hospital, R.M.P.H. Crolla

Elkerliek Hospital, J.A. Wegdam

Ikazia Hospital, W.J. Vles

Amstelveen Hospital, S.C. Veltkamp

Groene Hart Hospital, L.N.L. Tseng

Deventer Hospital, M.S.L. Liem

Hospital Group Twente, E.A. Kouwenhoven

Onze Lieve Vrouwe Gasthuis, M.F. Gerhards

Tergooi Hospitals location Hilversum 50, A. van Geloven

Rode Kruis Hospital, H.A. Cense

Mesos Medical Centre, A. Smits

Ropcke-Zweers Hospital, J.H. Tomee

Isala Clinics, G. A. Patijn

University MediCal Centre Groningen, B. van Leeuwen

Stichting Christelijke Zorgvoorzieningen, Talma Sionsberg, H. Bos

Diaconessen hospital Leiden, R. Vree

HagaHospital, W.H. Steup

Bronovo Hospital, F. van Duijnhoven

Gemini Hospital, N. Bode

St. Jansgasthuis Weert, N.A.J.B. Peters

Westfries Gasthuis, J.W. de Waard

Medical Centre Alkmaar, A.P.J. Houdijk

Orbis Medical Centre, J. Stoot

Medical Centre Leeuwarden, S.A. Koopal

Oosterschelde Hospitals, H. de Morree

University Medical Centre Utrecht, W.M.U. van Grevenstein

Heerenveen Hospital De Tjongerschans, F. Wit

Antonius Hospital Sneek, D. Hess

Spaarne Hospital, B. de Valk

Martini Hospital, W. Kelder
Canisius Wilhelmina Hospital, C. Rosman

\section{Author details}

'Department of Surgery, Jeroen Bosch Hospital, Nieuwstraat 34, 5211 NL 'sHertogenbosch, the Netherlands. ${ }^{2}$ Department of Surgery, Leiden University Medical Center, PO Box 9600, 2300 RC Leiden, the Netherlands. ${ }^{3}$ Department of Pathology, Jeroen Bosch Hospital, Nieuwstraat 34, 5211 NL 'sHertogenbosch, the Netherlands. ${ }^{4}$ Department of Pathology, Leiden University Medical Center, PO Box 9600, 2300 RC Leiden, the Netherlands. ${ }^{5}$ Department of Medical Oncology, Jeroen Bosch Hospital, Nieuwstraat 34, 5211 NL 's-Hertogenbosch, the Netherlands. ${ }^{6}$ Department of Medical Statistics, Leiden University Medical Center, PO Box 9600, 2300 RC Leiden, the Netherlands.

\section{Authors' contributions}

DJL drafted the manuscript. KB, CJHV, GJL edited the manuscript. All authors participated in the design of the study. HP performed the statistical analysis. All authors read and approved the final manuscript.

\section{Authors' information}

Daniel J. Lips', Boukje Koebrugge', Gerrit Jan Liefers ${ }^{2}$, Johannes C. van der Linden $^{3}$, Vincent T.H.B.M. Smit ${ }^{4}$, Hans F.M. Pruijt ${ }^{5}$, Hein Putter ${ }^{6}$, Cornelis J.H. van de Velde ${ }^{2}$, Koop Bosscha'.

${ }^{1}$ Department of Surgery, Jeroen Bosch Hospital, 's-Hertogenbosch, the Netherlands

${ }^{2}$ Department of Surgery, Leiden University Medical Center, Leiden, the Netherlands

${ }^{3}$ Department of Pathology, Jeroen Bosch Hospital, 's-Hertogenbosch, the Netherlands

${ }^{4}$ Department of Pathology, Leiden University Medical Center, Leiden, the Netherlands

${ }^{5}$ Department of Medical Oncology, Jeroen Bosch Hospital, 's-

Hertogenbosch, the Netherlands

${ }^{6}$ Department of Medical Statistics, Leiden University Medical Center, Leiden, the Netherlands

\section{Competing interests}

The EnRoute $\oplus$ Study is supported through an Educational Grant by Roche Pharmaceuticals.

The authors declare that they have no other competing interests.

Received: 3 October 2010 Accepted: 11 May 2011

Published: 11 May 2011

\section{References}

1. van Steenbergen LN, Lemmens VE, Louwman MJ, Straathof JW, Coebergh JW: Increasing incidence and decreasing mortality of colorectal cancer due to marked cohort effects in southern Netherlands. Eur J Cancer Prev 2009, 18:145-152.

2. Association of Comprehensive Cancer Centres: Guideline 'Colon cancer'. 2008 [http://www.oncoline.nl], Ref Type: Electronic Citation.

3. Nicastri DG, Doucette JT, Godfrey TE, Hughes SJ: Is occult lymph node disease in colorectal cancer patients clinically significant? A review of the relevant literature. J Mol Diagn 2007, 9:563-571.

4. Iddings D, Ahmad A, Elashoff D, Bilchik A: The prognostic effect of micrometastases in previously staged lymph node negative (NO) colorectal carcinoma: a meta-analysis. Ann Surg Oncol 2006, 13:1386-1392.

5. Bilchik A, Nissan A, Wainberg Z, Shen P, McCarter M, Protic M, et al: Surgical quality and nodal ultrastaging is associated with long-term disease-free survival in early colorectal cancer: an analysis of 2 international multicenter prospective trials. Ann Surg 2010, 252:467-474.

6. van der Zaag ES, Buskens CJ, Kooij N, Akol H, Peters HM, Bouma WH, et al: Improving staging accuracy in colon and rectal cancer by sentinel lymph node mapping: A comparative study. Eur I Surg Oncol 2009, 35:1065-1070.

7. van Schaik PM, van der Linden JC, Ernst MF, Gelderman WA, Bosscha K: Ex vivo sentinel lymph node "mapping" in colorectal cancer. Eur J Surg Oncol 2007, 33:1177-1182.

8. Stojadinovic A, Nissan A, Protic M, Adair CF, Prus D, Usaj S, et al: Prospective randomized study comparing sentinel lymph node evaluation with standard pathologic evaluation for the staging of colon 
carcinoma: results from the United States Military Cancer Institute Clinical Trials Group Study GI-01. Ann Surg 2007, 245:846-857.

9. Wong JH, Johnson DS, Namiki T, Tauchi-Nishi P: Validation of ex vivo lymphatic mapping in hematoxylin-eosin node-negative carcinoma of the colon and rectum. Ann Surg Oncol 2004, 11:772-777.

10. Nordgard O, Oltedal S, Korner H, Aasprong OG, Tjensvoll K, Gilje B, et al: Quantitative RT-PCR detection of tumor cells in sentinel lymph nodes isolated from colon cancer patients with an ex vivo approach. Ann Surg 2009, 249:602-607.

11. Sommariva A, Donisi PM, Gnocato B, Vianello R, Stracca PV, Zaninotto G: Factors affecting false-negative rates on ex vivo sentinel lymph node mapping in colorectal cancer. Eur J Surg Oncol 2010, 36(2):130-4.

12. de BM, van Deurzen CH, van Dijck JA, Borm GF, van Diest PJ, Adang EM, et al: Micrometastases or isolated tumor cells and the outcome of breast cancer. N Engl J Med 2009, 361:653-663.

13. Yun HR, Kim HC, Lee WY, Cho YB, Yun SH, Chun HK: The necessity of chemotherapy in T3NOMO colon cancer without risk factors. Am J Surg 2009, 198:354-358.

14. Morris M, Platell C, McCaul K, Millward M, van HG, Bayliss E, et al: Survival rates for stage II colon cancer patients treated with or without chemotherapy in a population-based setting. Int I Colorectal Dis 2007, 22:887-895.

15. Association of Comprehensive Cancer Centres. Association of Comprehensive Cancer Centers. 2010 [http://www.vikc.net], Ref Type: Electronic Citation

16. Figueredo A, Coombes ME, Mukherjee S: Adjuvant therapy for completely resected stage II colon cancer. Cochrane Database Syst Rev 2008, CD005390.

17. Efficacy of adjuvant fluorouracil and folinic acid in B2 colon cancer. International Multicentre Pooled Analysis of B2 Colon Cancer Trials (IMPACT B2) Investigators. J Clin Oncol 1999, 17:1356-63.

18. Gill S, Loprinzi CL, Sargent DJ, Thome SD, Alberts SR, Haller DG, et al: Pooled analysis of fluorouracil-based adjuvant therapy for stage II and III colon cancer: who benefits and by how much? J Clin Oncol 2004, 22:1797-1806.

19. O'Connell JB, Maggard MA, Ko CY: Colon cancer survival rates with the new American Joint Committee on Cancer sixth edition staging. J Natl Cancer Inst 2004, 96:1420-1425.

20. Andre T, Boni C, Mounedji-Boudiaf L, Navarro M, Tabernero J, Hickish T, et al: Oxaliplatin, fluorouracil, and leucovorin as adjuvant treatment for colon cancer. N Engl J Med 2004, 350:2343-2351.

21. Haller DG, Tabernero J, Maroun J, de Braud J, Price T, van cutsem E, et al: First efficacy findings from a randomized phase III trial of capecitabine + oxaliplatin vs. bolus 5-FU/LV for stage III colon cancer (NO16968/ XELOXA study). Eur J Cancer Supplements 2009, 7:4.

22. Taal BG, Van TH, Zoetmulder FA: Adjuvant 5FU plus levamisole in colonic or rectal cancer: improved survival in stage II and III. Br J Cancer 2001, 85:1437-1443.

23. Koyanagi K, Bilchik AJ, Saha S, Turner RR, Wiese D, McCarter M, et al: Prognostic relevance of occult nodal micrometastases and circulating tumor cells in colorectal cancer in a prospective multicenter trial. Clin Cancer Res 2008, 14:7391-7396.

24. Bilchik AJ, DiNome M, Saha S, Turner RR, Wiese D, McCarter M, et al: Prospective multicenter trial of staging adequacy in colon cancer: preliminary results. Arch Surg 2006, 141:527-533.

25. Saha S, Monson KM, Bilchik A, Beutler T, Dan AG, Schochet E, et al: Comparative analysis of nodal upstaging between colon and rectal cancers by sentinel lymph node mapping: a prospective trial. Dis Colon Rectum 2004, 47:1767-1772.

26. Saha $S$, Seghal R, Patel M, Doan K, Dan A, Bilchik A, et al: A multicenter trial of sentinel lymph node mapping in colorectal cancer: prognostic implications for nodal staging and recurrence. Am J Surg 2006, 191:305-310.

27. Cahill RA, Bembenek A, Sirop S, Waterhouse DF, Schneider W, Leroy J, et al: Sentinel node biopsy for the individualization of surgical strategy for cure of early-stage colon cancer. Ann Surg Oncol 2009, 16:2170-2180.

28. Bembenek AE, Rosenberg R, Wagler E, Gretschel S, Sendler A, Siewert JR, et al: Sentinel lymph node biopsy in colon cancer: a prospective multicenter trial. Ann Surg 2007, 245:858-863.
29. Kelder W, Braat AE, Karrenbeld A, Grond JA, De Vries JE, Oosterhuis JW, et al: The sentinel node procedure in colon carcinoma: a multi-centre study in The Netherlands. Int J Colorectal Dis 2007, 22:1509-1514.

30. Davies M, Arumugam PJ, Shah VI, Watkins A, Roger MA, Carr ND, et al: The clinical significance of lymph node micrometastasis in stage I and stage II colorectal cancer. Clin Transl Oncol 2008, 10:175-179.

31. Kobayashi H, Mochizuki H, Morita T, Kotake K, Teramoto T, Kameoka S, et al: Timing of relapse and outcome after curative resection for colorectal cancer: a Japanese multicenter study. Dig Surg 2009, 26:249-255.

32. Saad ED, Katz A, Hoff PM, Buyse M: Progression-free survival as surrogate and as true end point: insights from the breast and colorectal cancer literature. Ann Oncol 2009, 21(1):7-12.

33. Sargent DJ, Wieand HS, Haller DG, Gray R, Benedetti JK, Buyse M, et al: Disease-free survival versus overall survival as a primary end point for adjuvant colon cancer studies: individual patient data from 20,898 patients on 18 randomized trials. J Clin Oncol 2005, 23:8664-8670.

34. De GA, Hubbard J, Shi Q, O'Connell MJ, Buyse M, Benedetti J, et al: Association between disease-free survival and overall survival when survival is prolonged after recurrence in patients receiving cytotoxic adjuvant therapy for colon cancer: simulations based on the 20,800 patient ACCENT data set. J Clin Oncol 2010, 28:460-465.

\section{Pre-publication history}

The pre-publication history for this paper can be accessed here: http://www.biomedcentral.com/1471-2482/11/11/prepub

\section{doi:10.1186/1471-2482-11-11}

Cite this article as: Lips et al.: The influence of micrometastases on prognosis and survival in stage I-II colon cancer patients: the Enroute $\oplus$ Study. BMC Surgery 2011 11:11.

\section{Submit your next manuscript to BioMed Central and take full advantage of:}

- Convenient online submission

- Thorough peer review

- No space constraints or color figure charges

- Immediate publication on acceptance

- Inclusion in PubMed, CAS, Scopus and Google Scholar

- Research which is freely available for redistribution

Submit your manuscript at www.biomedcentral.com/submit
Ciomed Central 